\title{
First Commissioning Results from Hall $A$ at TJNAF
}

\author{
Robert Michaels \\ for the Hall A Collaboration
}

Thomas Jefferson National Accelerator Facility, Newport News, VA 23606, USA

\begin{abstract}
The commissioning and resulting capabilities of Hall A at the Thomas Jefferson National Accelerator Facility for performing coincidence experiments with a continuous-wave electron beam at energies of $0.8-6.0 \mathrm{GeV}$ will be described. The facility consists of a pair of high-resolution magnetic spectrometers to detect scattered particles, together with instrumentation to measure beam properties. The installation of the base equipment has been completed. Commissioning of the spectrometers, including their detector packages, is well advanced, both in single-arm mode and in coincidence mode.
\end{abstract}

Hall A at the Thomas Jefferson National Accelerator Facility is a new research facility for precise measurements of nucleon and nuclear structure at intermediate energies which will improve our understanding of the transition from low-energy phenomena to the regime where $\mathrm{pQCD}$ calculations are reliable. Jefferson Lab, with its continuous-wave beam of 0.8 to $6.0 \mathrm{GeV}$ and currents up to $100 \mu \mathrm{A}$, is ideal for coincidence measurements, where one spectrometer detects the scattered electron and the other spectrometer detects a knocked-out proton or other hadron. The experimental program currently has 29 approved experiments involving physicists from 34 institutions. This contribution reports on the first results of the Hall A Collaboration from the past year of commissioning the newly constructed spectrometers and instrumentation in Hall A. The first two experiments, involving coincidence knockout of protons from oxygen using a water target, are underway. Hall A is one of three experimental halls. Hall $\mathrm{B}$ is currently in a commissioning phase, while Hall $\mathrm{C}$ has been operational and taking data for about two years.

The Hall A facility consists of a pair of identical spectrometers of QQDQ design, together with detectors for detecting the scattered particles, beamline 
equipment, and targets (fig. 1). In order to achieve the physics aims, the spectrometers need to have a large acceptance, with excellent resolution and absolute accuracy in the reconstructed four-vectors of the events and precise normalization of the cross section. These requirements imply the need for a high resolution in the momentum measurement, which is achieved mainly by the large size of the magnet system. Good knowledge of the transfer matrix for the spectrometer is necessary to reconstruct the event at the scattering point. Also required are good pointing accuracy for the location of the spectrometers and precise measurements of beam properties such as position, angle, current, and charge integrated during an experiment.

The detector package consists of scintillators for triggering, vertical drift chambers for reconstruction of particle trajectories, and aerogel and Cherenkov detectors and lead glass arrays for particle ID. In addition, one of the spectrometers is outfitted with a focal plane polarimeter for measuring proton polarization, an apparatus reported on separately in this session. The trigger is formed in programmable CAMAC electronics and is configurable to include various combinations of the scintillator and particle ID detectors at the trigger level. The targets used in the initial operation have included carbon, beryllium oxide, and water.

Calibration of the optical transfer matrix for the spectrometers is performed in the following way. A $0.5 \mathrm{~cm}$ thick tungsten plate with a rectangular array of holes is placed at the entrance of the spectrometer. The matrix which transfers the tracks from the focal plane, where they are reconstructed in the drift chambers, to the target is determined through a chi-square minimization procedure which reproduces the hole pattern. In addition, the elastic peak from ${ }^{12} \mathrm{C}\left(\mathrm{e}, \mathrm{e}^{\prime}\right)$ is moved in steps across the focal plane, for small changes in the magnetic fields. The properties which have been obtained from the commissioning process are listed in the table below. Improvement in some of these properties may be expected in the upcoming year of operation.

Figure 2a shows the momentum spectrum of electrons elastically scattered from carbon, and the first few excited states, showing a momentum resolution of $3 \times 10^{-4}$ FWHM. Figure $2 \mathrm{~b}$ shows the missing-energy distribution for ${ }^{16} \mathrm{O}(\mathrm{e}, \mathrm{e}$ 'p) from a water target, showing a missing-energy resolution of about $1.3 \mathrm{MeV}$ FWHM. The missing-energy is defined as $E_{\text {miss }}=E-E^{\prime}-K_{\mathrm{p}}-K_{\mathrm{A}-1}$, where $E$ and $E^{\prime}$ are respectively the energy of the incoming and outgoing electron, and $K_{\mathrm{p}}$ and $K_{\mathrm{A}-1}$ are respectively the kinetic energies of the knocked-out proton and the residual nucleus. 
TABLE 1. Properties of Hall A and Spectrometers

\begin{tabular}{|l|l|}
\hline Luminosity & $\geq 10^{38} \mathrm{~cm}^{-2} \mathrm{sec}^{-1}$ \\
Momentum Range & $0.3-4.0 \mathrm{GeV} / \mathrm{c}$ \\
Momentum Acceptance & $\pm 4.5 \%$ \\
Momentum Resolution (FWHM) & $3 \times 10^{-4}$ \\
Solid Angle Acceptance & $6.5 \mathrm{msr}$ \\
Target Resolution (FWHM) & $4 \mathrm{~mm}$ \\
Coincidence Time Resolution (FWHM) & $1 \mathrm{~ns}$ \\
Missing Energy Resolution (FWHM) & $1.3 \mathrm{MeV}$ \\
\hline
\end{tabular}

During the next year, Hall A will continue the development of its capabilities, interleaved with experiments that can make use of existing capabilities. The Møller polarimeter for measuring beam polarization is presently being commissioned. A cryogenic target for liguid hydrogen and deuterium targets will be installed in August 1997. Two apparatus for measuring the beam energy with accuracy of $10^{-4}$ will be installed in the next year. A Compton polarimeter, for measuring the beam polarization at $1 \%$ accuracy, is planned for next year. 